\title{
Water-In-Oil Pickering Emulsions Stabilized by Water-Insoluble Polyphenol Crystals
}

\author{
Morfo Zembyla, Brent S. Murray, and Anwesha Sarkar*(D) \\ Food Colloids and Processing Group, School of Food Science and Nutrition, University of Leeds, Leeds LS2 9JT, U.K. \\ Supporting Information
}

\begin{abstract}
In recent years, there has been a resurgence of interest in Pickering emulsions because of the recognition of the unique high steric stabilization provided by particles at interfaces. This interest is particularly keen for water-in-oil (W/O) emulsions because of the limited range of suitable Pickering stabilizers available. We demonstrate for the first time that $\mathrm{W} / \mathrm{O}$ emulsions can be stabilized by using crystals from naturally occurring polyphenols (curcumin and quercetin particles). These particles were assessed based on their size, microstructure, contact angle, interfacial tension,

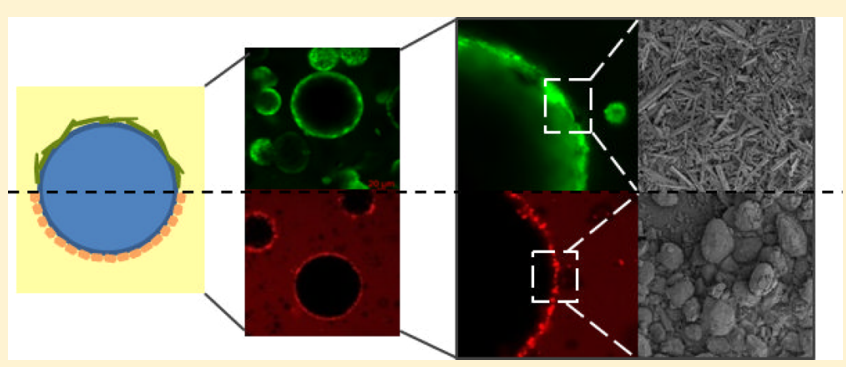
and $\zeta$-potential measurements in an attempt to predict the way that they act as Pickering stabilizers. Static light-scattering results and microstructural analysis at various length scales [optical microscopy, confocal laser scanning microscopy (CLSM), and scanning electron microscopy (SEM)] confirmed that the quercetin particles has a nearly perfect crystalline rod shape with a high aspect ratio; that is, the ratio of length to diameter $(L / D)$ was ca. $2.5: 1-7: 1$. On the other hand, the curcumin particles $\left(d_{3,2}=0.2 \mu \mathrm{m}\right)$ had a polyhedral shape. Droplet sizing and CLSM revealed that there was an optimum concentration $(0.14$ and $0.25 \mathrm{wt} \%$ for quercetin and curcumin, respectively) where smaller water droplets were formed $\left(d_{3,2} \approx 6 \mu \mathrm{m}\right)$. Interfacial shear viscosity $\left(\eta_{i}\right)$ measurements confirmed that a stronger film was formed at the interface with quercetin particles $\left(\eta_{i} \approx 25 \mathrm{~N} \mathrm{~s}\right.$ $\left.\mathrm{m}^{-1}\right)$ rather than with curcumin particles $\left(\eta_{i} \approx 1.2 \mathrm{~N} \mathrm{~s} \mathrm{~m}^{-1}\right)$ possibly because of the difference in the shape and size of the two crystals. This study provides new insights into the creation of Pickering W/O emulsions with polyphenol crystals and may lead to various soft matter applications where Pickering stabilization using biocompatible particles is a necessity.
\end{abstract}

\section{INTRODUCTION}

Water-in-oil (W/O) emulsions are widely used for different soft matter applications in food, pharmaceutical, personal care, agriculture, and so forth. Recently, the use of Pickering particles as stabilizers for emulsions has gained significant attention owing to their ability to adsorb irreversibly at the liquid/liquid interface. ${ }^{1,2}$ Pickering stabilization arises when particles accumulate at the water-oil $(\mathrm{W} / \mathrm{O})$ interface forming a mechanical (steric) barrier that protects the emulsion droplets against coalescence. ${ }^{3}$ Effective Pickering stabilization is achieved when the average size of the adsorbed particles is at least an order of magnitude smaller than the emulsion droplet size. $^{4,5}$

The size of the particles dispersed in the continuous phase is an important parameter that determines the Pickering functionality. Simple geometrical consideration can be used to estimate the minimum amount of surface-active particles required for adequate surface coverage in order to form stable emulsions. ${ }^{6}$ The particle contact angle $(\theta)$ represents the wettability of a particle at the interface, identifying whether the particle preferentially resides in the water or oil phase. ${ }^{7}$ If $\theta$, measured in the aqueous phase, is smaller than $90^{\circ}$, a larger fraction of the particle surface will reside in the aqueous rather than in the nonpolar phase; that is, the particle is more hydrophilic and will tend to stabilize an $\mathrm{O} / \mathrm{W}$ emulsion.
However, if $\theta$ is greater than $90^{\circ}$, the particle will reside more in the oil phase; that is, the particle is more hydrophobic and will stabilize $\mathrm{W} / \mathrm{O}$ emulsions. Maximum adsorption occurs when particles are wetted equally by oil and water and have a contact angle of exactly $90^{\circ} .7,8$ The stability of Pickering emulsions is usually inversely proportional to the particle size; smaller particles will give a higher packing efficiency and therefore a more homogeneous layer at the interface preventing coalescence. ${ }^{9,10}$ Particle size also has a direct effect on the energy of desorption $\left(\Delta G_{\mathrm{d}}\right)$, and if adsorption occurs, smaller particles provide lower $\Delta G_{\mathrm{d}}$, according to eq 1 as follows:

$$
\Delta G_{\mathrm{d}}=\pi r^{2} \gamma_{\mathrm{ow}}(1 \pm|\cos (\theta)|)^{2}
$$

where $\gamma_{\text {ow }}$ is the interfacial tension between the oil and water phases, $|\cos (\theta)|$ is the modulus of $\cos (\theta), \theta$ is the particle contact angle (the sign inside the bracket is negative if the particles are removed into the water phase and positive if removed into the oil phase), and $r$ is the particle radius, that is, assumed spherical. Thus, smaller particles can more easily detach from the $\mathrm{W} / \mathrm{O}$ interface than larger ones. ${ }^{10}$ However, it

Received: May 4, 2018

Revised: June 25, 2018

Published: August 3, 2018 
should be noted that even particles with just a few $10 \mathrm{~s}$ of nanometer in radius can have a $\Delta G_{\mathrm{d}}$ of several $1000 k_{\mathrm{B}} T$ at 298 $\mathrm{K}^{5}$ Note that this assumes that the particles are perfectly spherical, and thus, eq 1 is rarely directly applicable in real systems. Nonspherical particles of the same volume and the same material as spherical particles will have a greater specific surface area and therefore higher desorption energies than the equivalent spherical particles. ${ }^{11}$

Mostly, synthetic particles, such as hydrophobic silica, modified cellulose, polystyrene latex, microgels from crosslinked poly( $\mathrm{N}$-isopropylacrylamide)-co-methacrylic acid, and so forth, have been used in the literature to stabilize W/O emulsions. ${ }^{12-17}$ Therefore, there is an enormous opportunity to identify a new class of particles for the stabilization of W/O emulsions where the particles are based on natural, biodegradable, and renewable resource ingredients that are immediately suitable for soft matter applications (food, pharmaceuticals, cosmetics, agrochemicals, etc.), for which biocompatibility is a key requirement. In this regard, polyphenol crystals would be promising biocompatible Pickering stabilizers. Polyphenol molecules have the characteristics of a particular plant species and even particular organs or tissues of that plant species and have received significant attention in recent years because of their reported biological activities and widespread occurrence in the diet. ${ }^{18}$ Fruits, vegetables, leaves, seeds, and other types of foods and beverages such as tea, chocolate, and wine are rich sources of polyphenols. ${ }^{19}$

Luo et al. ${ }^{6}$ suggested for the first time that polyphenol crystals may be adsorbed at the liquid-liquid interface. The authors identified that some common polyphenols can act as stabilizers for $\mathrm{O} / \mathrm{W}$ emulsions through their adsorption as water-insoluble particles to the surface of the oil droplets. It was demonstrated that polyphenols with different $\log P$ values, where $P$ is the partition coefficient between $n$-octanol and water, can act as Pickering stabilizers at the $\mathrm{O} / \mathrm{W}$ interface, stabilizing emulsions for several weeks without any obvious visual changes. ${ }^{6}$ The $\log P$ value can be used to identify the hydrophilicity of a polyphenol molecule. However, Pickering stabilization depends on the hydrophilic/hydrophobic balance of the particles, not their molecules, and no simple relationship seemed to exist between $\log P$ and the ability to stabilize $\mathrm{O} / \mathrm{W}$ emulsions. More importantly, here the ability of polyphenol crystals to stabilize $\mathrm{W} / \mathrm{O}$ emulsions apparently still remains uninvestigated.

In this study, quercetin and curcumin particles were investigated for their potential to act as Pickering stabilizers for W/O emulsions because of their widespread availability, "clean-label" feature, and biocompatibility. Curcumin is a natural low-molecular-weight polyphenolic compound found in the rhizomes of the perennial herb turmeric (Curcuma longa). ${ }^{20}$ From a structural viewpoint, curcumin (Figure 1a)

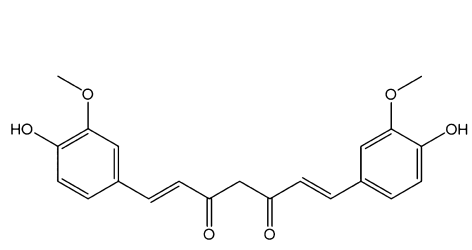

(a)

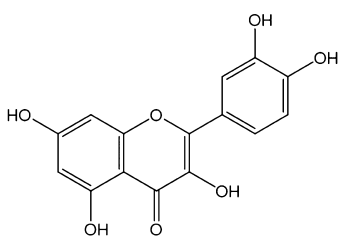

(b)
Figure 1. Structures of curcumin (a) and quercetin (b) molecules. comprises two aromatic rings with methoxyl and hydroxyl groups in the ortho position with respect to each other. ${ }^{21}$ The aromatic rings are connected through seven carbons that contain two $\alpha, \beta$-unsaturated carbonyl groups. ${ }^{21}$ As a result, curcumin exists in three possible forms, two isomers in an equilibrating keto-enol tautomeric form and the $\alpha, \beta$-diketonic tautomeric form. ${ }^{22}$ The polar hydroxyl/ketone groups are expected to impact hydrophilicity, whereas the aromatic/ aliphatic parts would be expected to make the molecule more hydrophobic. Quercetin is a flavonoid characterized by its C6C3-C6 basic backbone and is found in onions, kale, French beans, broccoli, and so forth. ${ }^{23}$ Quercetin (Figure 1b) has hydroxyl groups that impart hydrophilic characteristics, whereas the ring structures impact the hydrophobicity; thus, it can be described as an amphiphilic molecule. ${ }^{6}$ Both curcumin and quercetin are relatively insoluble in water with high $\log P$ values of 3.29 and 2.16, respectively, and appear to be relatively insoluble in vegetable oils. The maximum solubility of curcumin and quercetin in vegetable oils has been reported to be 0.02 and $\sim 0.017 \mathrm{wt} \%$, respectively, which suggests that these materials would have been predominantly in their insoluble particle (crystal) form in the soybean oil. ${ }^{24,25}$ First, the interfacial properties of these particles dispersed in oil were investigated in terms of their size, wettability, and interfacial behavior to assess their potential as Pickering stabilizers. Second, the characteristics of $\mathrm{W} / \mathrm{O}$ emulsions stabilized by curcumin or quercetin particles were evaluated using a range of complimentary physical and microstructural techniques at different $\mathrm{pH}$ values. To our knowledge, the present study is the first report of the use of polyphenol crystals to stabilize $\mathrm{W} / \mathrm{O}$ emulsions.

\section{MATERIAL AND METHODS}

Materials. Curcumin (an orange-yellow powder) from turmeric rhizome (95 wt \% total curcuminoid content) was obtained from Alfa Aesar (UK). Quercetin $(\geq 95 \%)$ in the form of a yellow crystalline solid was purchased from Cayman Chemicals (USA). Both polyphenols were used without further purification. Soybean oil (KTC Edibles, UK) was purchased from a local store. Aluminum oxide, $99 \%$, extra pure, was used for soybean oil purification in some experiments and was purchased from Acros Organics (Belgium). Water purified by treatment with a Milli- $Q$ apparatus (Millipore, Bedford, UK) with a resistivity not less than $18 \mathrm{M} \Omega \mathrm{cm}$ was used for the preparation of emulsions. A few drops of hydrochloric acid $(0.1 \mathrm{M}$ $\mathrm{HCl})$ or sodium hydroxide $(0.1 \mathrm{M} \mathrm{NaOH})$ were used to adjust the $\mathrm{pH}$ of the emulsions. Sodium azide was purchased from SigmaAldrich (USA).

\section{EXPERIMENTAL SECTION}

Contact Angle and Wettability. The hydrophilic/hydrophobic character of the polyphenol crystals was evaluated in terms of their wettability. The wettability measurements were carried out at $25{ }^{\circ} \mathrm{C}$ using a OCA25 drop-shape tensiometer (DataPhysics Instruments, Germany) fitted with a microsyringe and a high-speed camera. Compressed discs of the particulate materials were prepared by placing $0.3 \mathrm{~g}$ of curcumin or quercetin crystals between the plates of a hydraulic bench press (Clarke, UK) using a $1.54 \mathrm{~cm}$ diameter die under a weight of 6 tonnes for $30 \mathrm{~s}$. Static contact angles were measured using the sessile drop method. Water or oil droplets $(3 \mu \mathrm{L})$ were produced using a straight needle of $0.52 \mathrm{~mm}$ outer diameter and $0.26 \mathrm{~mm}$ internal diameter, to form a sessile drop onto the compressed particle disc surfaces. A video camera was used to record the droplet behavior. The droplet contour was fitted using the SCA software, and the contact angles between the particle substrate and the water $\left(\theta_{\mathrm{w}}\right)$ or oil droplet $\left(\theta_{\mathrm{o}}\right)$ were measured. All measurements 
were carried out in triplicate, and error bars represent the standard deviations.

Interfacial Tension. The interfacial tension $(\gamma)$ measurements were performed between the soybean oil with or without the presence of polyphenol crystals and Milli- $\mathrm{Q}$ water $(\mathrm{pH} 3)$ using the pendant drop method in a DataPhysics OCA tensiometer (DataPhysics Instruments, Germany). The apparatus includes an experimental cell, an optical system for the illumination and the visualization of the drop shape, and a data acquisition system. An upward bended needle was used to immerse a drop of a lower density liquid into a higher density one. Thus, a drop of soybean oil or oil suspension (0.14 wt \% curcumin or quercetin dispersed in soybean oil) was formed at the tip of the needle and suspended in the cuvette containing Milli- $Q$ water, at $\mathrm{pH}$ 3. The contour of the drop extracted by the SCA 20 software was fitted to Young-Laplace equation to obtain $\gamma$. All measurements were carried out in triplicate, and error bars represent the standard deviations.

Preparation of Oil Dispersions and W/O Emulsions. Curcumin or quercetin dispersions were prepared by dispersing these polyphenol crystals in the continuous phase (soybean oil) using an Ultra-Turrax T25 mixer (Janke \& Kunkel, IKA-Labortechnik) with a $13 \mathrm{~mm}$ mixer head (S25N-10 G) operating at $9400 \mathrm{rpm}$ for $5 \mathrm{~min}$. The aqueous phase was Milli- $\mathrm{Q}$ water at $\mathrm{pH} 3$. The $\mathrm{pH}$ of the water was adjusted by adding few drops of $0.1 \mathrm{M} \mathrm{HCl}$, and $0.02 \%$ sodium azide was added into the aqueous phase as a preservative. Coarse emulsions were prepared by homogenizing 5 wt $\%$ of the aqueous phase with 95 wt \% of the oil phase using an Ultra-Turrax mixer for 2 $\mathrm{min}$ at $13400 \mathrm{rpm}$. Fine emulsions were prepared by passing the coarse emulsions through a high-pressure Leeds jet homogenizer, ${ }^{26}$ twice, operated at 300 bar. The initial temperature of the particle dispersion was $21{ }^{\circ} \mathrm{C}$. The temperature of the dispersion was 23 and $26^{\circ} \mathrm{C}$ after Ultra-Turrax mixing at $9400 \mathrm{rpm} / 5 \mathrm{~min}$ and $13400 \mathrm{rpm} /$ $2 \mathrm{~min}$, respectively. The temperature of particle dispersion after passing through the jet homogenizer (two passes) was $33-34{ }^{\circ} \mathrm{C}$. These slight temperature increases were too low to have any significant impact on the solubility of the particles. Immediately after preparation, the emulsions were sealed in $25 \mathrm{~mL}$ cylindrical tubes (internal diameter $=17 \mathrm{~mm}$ ) and stored at room temperature in a dark place.

Particle and Emulsion Droplet Size Measurements. Particle and emulsion droplet size distributions were measured using static light scattering (SLS) via a Mastersizer Hydro SM small-volume wet sample dispersion unit (Malvern Instruments, UK). Average droplet size was monitored via the Sauter mean diameter, $d_{3,2}$, or the volume mean diameter, $d_{4,3}$, defined by

$$
d_{\mathrm{ab}}=\frac{\sum n_{i} d_{i}^{\mathrm{a}}}{\sum n_{i} d_{i}^{\mathrm{b}}}
$$

where $n_{i}$ is the number of droplets of diameter $d_{i}$.

Refractive indices of $1.42,1.82$, and 1.47 for curcumin, quercetin, and soybean oil were used, respectively, for particle size measurements. For size measurements of water droplets, refractive indices of 1.33 and 1.47 were used for water and soybean oil, respectively. Absorption coefficients of $0.01,0.1$, and 0.01 for curcumin, quercetin, and water were used, respectively. All measurements were made at room temperature on at least three different samples.

$\zeta$-Potential Measurements. The $\zeta$-potential measurements of curcumin and quercetin dispersions in water at $\mathrm{pH} 3$ and 7 values were performed using a Nanoseries ZS instrument (Zetasizer NanoZS, Malvern Instruments, Worcestershire, UK). The instrument software was used to convert the electrophoretic mobility into $\zeta$ potential values using the Smoluchowski assumption (see eq 3 ). Polyphenol dispersions were prepared by mixing the polyphenol crystals $(0.14$ wt \%) with water at $\mathrm{pH} 3$ or 7 , via an Ultra-Turrax mixer operating at $9400 \mathrm{rpm}$ for $5 \mathrm{~min}$. The $\mathrm{pH}$ of freshly prepared suspensions was adjusted at $\mathrm{pH} 3$ and 7 using $0.1 \mathrm{M} \mathrm{HCl}$ or $\mathrm{NaOH}$. Three readings of $\zeta$-potential were made per sample.

The $\zeta$-potential was calculated from the measured electrophoretic mobility using the Henry equation ${ }^{27}$

$$
U_{\mathrm{E}}=\frac{2 \varepsilon \zeta f(k a)}{3 \eta}
$$

where $U_{\mathrm{E}}$ is the electrophoretic mobility, $\zeta$ is the zeta-potential, $\varepsilon$ is the dielectric constant of water, $\eta$ is the viscosity of water, and $f(k a)$ is Henry's function, assumed equal to 1.5 using the Smoluchowski approximation. It should be noted that although in $\mathrm{W} / \mathrm{O}$ emulsions the particles were dispersed in oil, we cannot use this equipment for measuring the $\zeta$-potential in such nonaqueous systems. However, it was expected that the $\zeta$-potential measured in the aqueous phase might provide some insights into the behavior of the crystals when partially wetted by water at the $\mathrm{W} / \mathrm{O}$ interface.

Optical Microscopy. A drop of the curcumin or quercetin dispersion $(0.14$ wt $\%)$ was placed on a microscope slide and then covered with a cover slip $(0.17 \mathrm{~mm}$ thickness). The size and shape of the polyphenol crystals were observed using a liquid-crystal display digital microscope (PentaView, Celestron, USA). The samples were scanned at room temperature $\left(25 \pm 1{ }^{\circ} \mathrm{C}\right)$ using $20 \times / 0.4$ objective lenses.

Scanning Electron Microscopy. The particle morphology of the curcumin and quercetin crystals was imaged using a scanning electron microscope (Nova NanoSEM450, FEI, USA). Curcumin or quercetin dispersions were prepared as described above and filtered through 0.20 or $0.45 \mu \mathrm{m}$ filter papers, respectively. The particles on the filter paper were left overnight in a vacuum oven at $25^{\circ} \mathrm{C}$. Then, they were coated with iridium $(4 \mathrm{~nm})$ and analyzed using a $2 \mathrm{keV}$ beam to prevent damage to the sample.

Confocal Laser Scanning Microscopy. The microstructure of the $\mathrm{W} / \mathrm{O}$ emulsion droplets was observed using a confocal microscope (Zeiss LSM700 inverted, Germany). The emulsions were gently agitated before analysis to ensure that a homogeneous sample was observed. Approximately $80 \mu \mathrm{L}$ of the samples was placed into a laboratory-made welled slide and a cover slip $(0.17 \mathrm{~mm}$ thickness) was placed on top, ensuring that there was no air gap (or bubbles) trapped between the sample and the coverslip. The samples were scanned at room temperature $\left(25 \pm 1{ }^{\circ} \mathrm{C}\right)$ using $20 \times / 0.5$ objective lens. Autofluorescence from the particles was excited using 488 and $405 \mathrm{~nm}$ lasers for curcumin and quercetin crystals, respectively.

Interfacial Shear Viscosity $\left(\boldsymbol{\eta}_{i}\right)$. A two-dimensional Couette-type interfacial viscometer ${ }^{28,29}$ was operated in a constant shear rate mode. A stainless steel biconical disc (radius $15.0 \mathrm{~mm}$ ) was suspended from a thin torsion wire with its edge in the plane of the W/O interface of the solution contained within a cylindrical glass dish (radius 72.5 $\mathrm{mm}$ ). For these measurements, 0.14 wt $\%$ curcumin and quercetin particles were dispersed in purified or nonpurified oil using the UltraTurrax mixer at $9400 \mathrm{rpm}$ for $5 \mathrm{~min}$. For the experiments with purified oil, the oil was purified with aluminum oxide to eliminate free fatty acids and surface-active impurities that may affect the measurements. A mixture of oil and aluminum oxide in a proportion of $2: 1 \mathrm{w} / \mathrm{w}$ was stirred for $3 \mathrm{~h}$ and centrifuged at $4000 \mathrm{rpm}$ for 30 min.

The constant shear rate apparent interfacial viscosity, $\eta_{i}$, is given by the following equation:

$$
\eta_{i}=\frac{g_{\mathrm{f}}}{\omega} K\left(\theta-\theta_{\mathrm{o}}\right)
$$

where $K$ is the torsion constant of the wire, $\theta$ is the equilibrium deflection of the disc in the presence of the film, $\theta_{\mathrm{o}}$ is the equilibrium deflection in the absence of the film, that is, due to the bulk drag of the sub-phase on the disc, $g_{\mathrm{f}}$ is the geometric factor, and $\omega$ is the angular velocity of the dish. A fixed value of $\omega=1.27 \times 10^{-3} \mathrm{rad} \mathrm{s}^{-1}$ was used.

\section{RESULTS AND DISCUSSION}

Assessment of Polyphenol Crystals as Pickering Stabilizers. Size and Shape of Curcumin and Quercetin Crystals Dispersed in Oil. In this study, the size of the curcumin and quercetin crystals dispersed in soybean oil was 
measured after treatment with the Ultra-Turrax mixer at 9500 $\mathrm{rpm}$ for $5 \mathrm{~min}$ as shown in Figure 2. The particle size

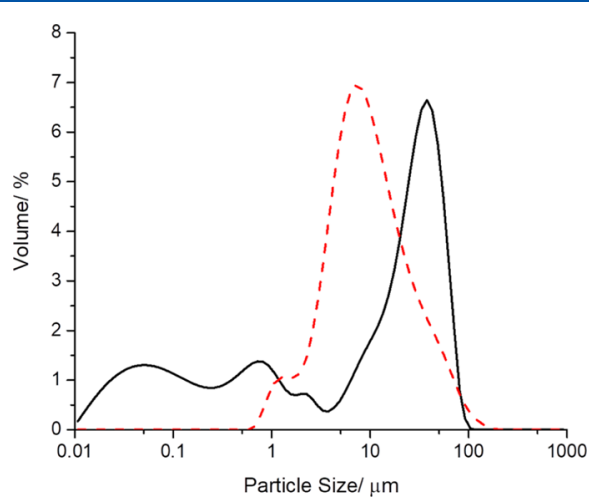

Figure 2. Particle size distribution of curcumin (solid black line, 0.14 wt \%) and quercetin (red dashed line, 0.14 wt \%) particles dispersed in soybean oil.

distribution shows that curcumin particles were polydisperse with $d_{3,2}$ and $d_{4,3}$ values of 0.2 and $20.5 \mu \mathrm{m}$, respectively. In comparison, quercetin particles were more monodisperse with $d_{3,2}$ and $d_{4,3}$ values of 5.9 and $14.9 \mu \mathrm{m}$, respectively. The sizes of the particles passed through all the stages of the homogenization process (Ultra Turrax $9400 \mathrm{rpm} / 5 \mathrm{~min}, 13$ $400 \mathrm{rpm} / 2 \mathrm{~min}$ and jet homogenizer, two passes, at $300 \mathrm{bar}$ ) are shown in the Supporting Information, Figure S1 and Table S1. The jet homogenizer produced a slight milling effect, but this appeared to predominantly affect the larger crystals or their aggregates $(>10 \mu \mathrm{m})$. The overall particle distributions, which were quite broad, still remained largely in the micrometer range and were comparable with Figure 2, although Figure 2 also shows that the curcumin part of the distribution extends to smaller sizes compared to the quercetin part.

The microstructure of curcumin (Figure 3) and quercetin (Figure 4) dispersions in soybean oil was observed using optical microscopy, confocal laser scanning microscopy (CLSM), and scanning electron microscopy (SEM) at

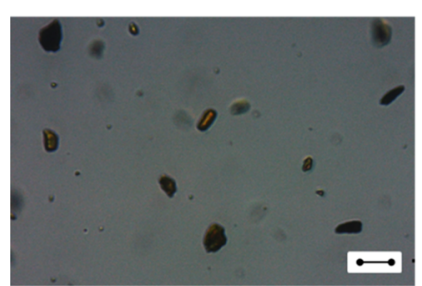

(a)

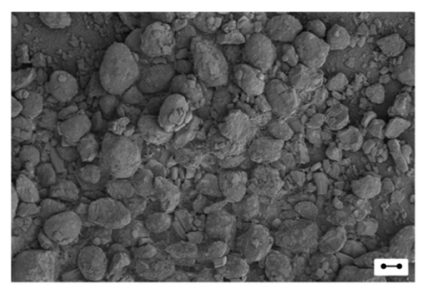

(c)

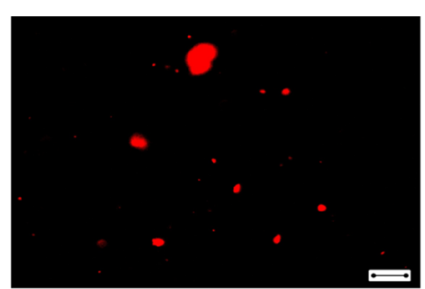

(b)

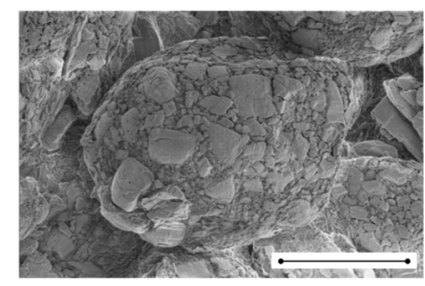

(d)
Figure 3. Microstructure of curcumin dispersion ( 0.14 wt \%) in soybean oil at various length scales: optical microscopy (a), CLSM at $488 \mathrm{~nm}$ excitation (b), SEM at lower magnification $(250 \times)(\mathrm{c})$, and SEM at higher magnification $(2000 \times)(d)$. Scale bar is $20 \mu \mathrm{m}$.

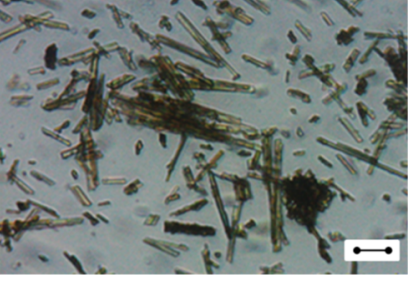

(a)

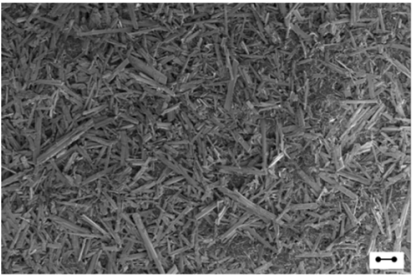

(c)

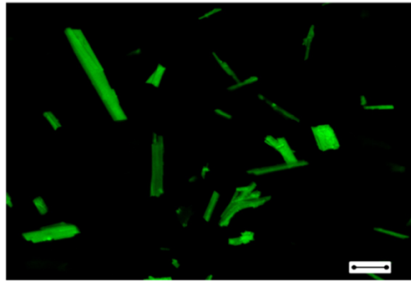

(b)

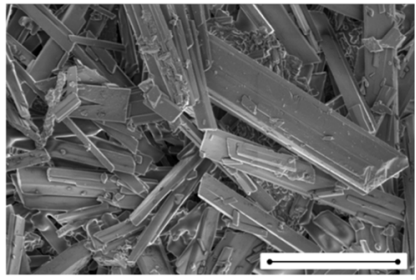

(d)
Figure 4. Microstructure of quercetin dispersion ( $0.14 \mathrm{wt} \%)$ in soybean oil at various length scales: optical microscopy (a), CLSM at $405 \mathrm{~nm}$ excitation (b), SEM at lower magnification $(250 \times)(\mathrm{c})$, and SEM at higher magnification $(2000 \times)(d)$. Scale bar is $20 \mu \mathrm{m}$.

different magnifications. The curcumin particles appeared to have a rather polyhedral shape, whereas the quercetin particles had a nearly perfect microcrystalline structure with a rod shape. The results from the size distribution plots measured using the Mastersizer (Figure 2) can be confirmed from the figures of curcumin (Figure $3 a-c$ ) and quercetin (Figure $4 a-c$ ) particles at lower magnification. For curcumin particles, there was a range of different sizes $(0.5-15 \mu \mathrm{m})$; however, for quercetin, the particles had a length of $5-35 \mu \mathrm{m}$ and a ratio of length to diameter $(L / D)$ of around 2.5:1-7:1. At higher magnification for both curcumin (Figure $3 \mathrm{~d}$ ) and quercetin (Figure $4 \mathrm{~d}$ ) particles, it was clear that the particles were aggregates of smaller particles, probably because of the filtration used on the preparation procedure. The microstructure of curcumin and quercetin powders was observed using SEM at different magnifications (see Supporting Information, Figure S2). It can been observed that the shape of the crystals in the powdered form was very similar to that of the ones dispersed in oil (Figures $3 c, d$ and $4 c, d$ ), indicating that there was no change of the crystal habit when they were passed through the stages of the emulsification process.

Contact Angle and Wettability of Polyphenol Particles. The hydrophilic/hydrophobic character of particles can be identified via their wettability (the tendency of one liquid to spread on a solid surface) in aqueous and oil phases. ${ }^{10}$ When the contact angle of a water droplet, $\theta_{w}$, significantly exceeds the contact angle of an oil droplet, $\theta_{\mathrm{o}}$, the particles can be categorized as hydrophobic. In other words, they are preferentially wetted by oil and will tend to stabilize $\mathrm{W} / \mathrm{O}$ rather than $\mathrm{O} / \mathrm{W}$ emulsions. ${ }^{30}$

In this study, both curcumin and quercetin particles had $\theta_{\mathrm{w}}$ values exceeding their $\theta_{\mathrm{o}}$ values, indicating that both possess a hydrophobic character as shown in Table 1. For curcumin particles, $\theta_{\mathrm{w}}$ at $\mathrm{pH} 3$ was significantly larger than that at $\mathrm{pH} 7$. This agrees with Tønnesen et al. ${ }^{31}$ that the curcumin molecule is hydrophobic with a very low water solubility $(11 \mathrm{ng} / \mathrm{mL}$ at $25{ }^{\circ} \mathrm{C}$ ) because of its aliphatic chains. ${ }^{32}$ Quercetin particles had a lower $\theta_{\mathrm{w}}$ than curcumin particles, probably because of the presence of more $-\mathrm{OH}$ groups on their backbone and the formation of hydrogen bonds with water molecules. Quercetin 
Table 1. Measured Contact Angles of Water Droplets (pH 3 and 7) on Polyphenol Crystals in Air ( $\theta_{\mathrm{w}}$ ) and Oil Droplets on Polyphenol Crystals in Air $\left(\theta_{0}\right)$; Also Shown Is the Calculated Three-Phase Contact Angle $(\theta)$ at the W/O Interface in the Aqueous Phase (pH 3 and 7$)^{a}$

\begin{tabular}{|c|c|c|c|c|c|}
\hline \multicolumn{4}{|c|}{ contact angle at the polyphenol, water, or oil and air interface/deg } & \multicolumn{2}{|c|}{ three-phase contact angle/deg } \\
\hline \multirow[b]{2}{*}{ particle type } & \multicolumn{2}{|c|}{ aqueous phase $\left(\theta_{\mathrm{w}}\right)$} & \multirow[b]{2}{*}{ oil phase $\left(\theta_{\mathrm{o}}\right)$} & \multicolumn{2}{|c|}{ aqueous phase } \\
\hline & $\mathrm{pH} 3$ & $\mathrm{pH} 7$ & & $\mathrm{pH} 3$ & $\mathrm{pH} 7$ \\
\hline curcumin & $85.6 \pm 0.4$ & $73.4 \pm 1.2$ & $11.9 \pm 2.0$ & $175.3 \pm 1.0$ & $115.7 \pm 2.1$ \\
\hline quercetin & $60.2 \pm 0.7^{\mathrm{a}}$ & $59.4 \pm 0.4^{\mathrm{a}}$ & $19.8 \pm 0.5$ & $80.9 \pm 2.1^{\mathrm{b}}$ & $79.1 \pm 1.4^{\mathrm{b}}$ \\
\hline
\end{tabular}

${ }^{a}$ Samples with the same letter do not differ significantly $(p>0.05)$ according to Tukey's test.

crystal structure can be described as layers of hydrogen bond dimers. These dimers form a two-dimensional net connected through a hydrogen bonding network with water molecules present in the quercetin. ${ }^{33}$ Therefore, quercetin molecules can pack in a way that some of the $-\mathrm{OH}$ groups are not exposed to the continuous phase, giving a hydrophobic nature to the particle surface. It can be observed that the different $\mathrm{pH}$ values did not affect the contact angle on the quercetin particles as much as for curcumin. The same effect was observed for the oil phase.

From these values, we estimated the three-phase contact angle $(\theta)$ of the polyphenol particles at the W/O interface via Young's equation (see Table 1 ). We used measured values of $70.8,32.5$, and $26.4 \mathrm{mN} / \mathrm{m}$ for 0.14 wt \% curcumin in oil and $71.5,33.4$, and $26.4 \mathrm{mN} / \mathrm{m}$ for $0.14 \mathrm{wt} \%$ quercetin in oil for the $\mathrm{A} / \mathrm{W}, \mathrm{A} / \mathrm{O}$, and $\mathrm{W} / \mathrm{O}$ interfacial tensions, respectively (see Supporting Information, Table S2). Note that the A/W tensions were measured after contacting the oil phase with water and are very close to the value for pure water $(72.0 \mathrm{mN} /$ $\mathrm{m})$, suggesting little solubilization of surface-active components from the oil into the aqueous phase. Similarly, the W/O tension value measured for purified soybean oil without polyphenols was $\sim 32 \mathrm{mN} / \mathrm{m}$ (see Supporting Information, Table S3), only slightly higher than the value of $\sim 26 \mathrm{mN} / \mathrm{m}$ measured in the presence of curcumin or quercetin (in nonpurified oil). This suggests limited solubilization of the polyphenol crystals in the oil and their molecular adsorption at the $\mathrm{W} / \mathrm{O}$ interface. The calculated $\theta$ values are 175 and $116^{\circ}$ for curcumin at $\mathrm{pH} 3$ and 7 , respectively, and 81 and $79^{\circ}$ for quercetin at $\mathrm{pH} 3$ and 7 , respectively. Although one should view these values with some caution because the components from the oil could still adsorb to the surfaces of the crystals and affect their microscopic contact angles. It is interesting to apply these values to eq 1 and calculate possible $\Delta G_{\mathrm{d}}$. Assuming a spherical particle of radius $0.1 \mu \mathrm{m}$, one obtains values of $8.0 \times$ $10^{5}$ and $4.2 \times 10^{5} k_{\mathrm{B}} T$ for curcumin at $\mathrm{pH} 3$ and 7 , respectively, and $2.7 \times 10^{5}$ and $2.9 \times 10^{5} k_{\mathrm{B}} T$ for quercetin at $\mathrm{pH} 3$ and 7, respectively. Note that for $r=1 \mu \mathrm{m}$ (closer to the size of the majority of the particles observed), all these values would be $100 \times$ higher, confirming that there is a very strong driving force for the crystals to get trapped at the interface. The $\theta$ and $\Delta G_{\mathrm{d}}$ calculations therefore confirm the above discussion of the relative hydrophobicities of the crystals based on just their water/solid and oil/solid contact angles (Table 1). The relatively small lowering of the $\mathrm{W} / \mathrm{O}$ interfacial tension in the presence of the particles is also another characteristic of Pickering stabilization (see Supporting Information, Table S3)..$^{34,35}$

$\zeta$-Potential Measurements. The surface charge and therefore the $\zeta$-potential of particles are frequently $\mathrm{pH}$-dependent and can potentially affect particle dispersion/aggregation. ${ }^{30}$ It was not possible to measure the $\zeta$-potential of particles dispersed in soybean oil. Therefore, in order to predict how the particles might behave when they adsorb at the W/O interface, the $\zeta$-potential of those particles dispersed in the aqueous phase was measured because this might have some relevance to the behavior of the particles when partially wetted by water at the $\mathrm{W} / \mathrm{O}$ interface. Table 2 shows the $\zeta$-potential of curcumin

Table 2. $\zeta$-Potential $(\mathrm{mV})$ of Curcumin and Quercetin Particles (0.14 wt \%) Dispersed in the Aqueous Phase at $\mathrm{pH}$ 3 and $7^{a}$

$\begin{array}{ccc}\text { particle type } & \mathrm{pH} 3 & \mathrm{pH} 7 \\ \text { curcumin } & 12.0 \pm 2.2 & -47.6 \pm 2.4^{*} \\ \text { quercetin } & -26.2 \pm 1.2 & -48.1 \pm 1.2^{*}\end{array}$

${ }^{a}$ Samples with $*$ do not differ significantly $(p>0.05)$ according to Tukey's test.

and quercetin particles dispersed in water at $\mathrm{pH} 3$ and 7. The curcumin particles had a positive charge at $\mathrm{pH} 3$, but at $\mathrm{pH} 7$, they became strongly anionic. Under alkaline conditions, curcumin can be dissolved sparsely in water as the acidic phenolic group in curcumin donates its $\mathrm{H}^{+}$ion, forming the phenolate ion enabling dissolution. ${ }^{21}$ On the other hand, quercetin had negative charge at both $\mathrm{pH}$ values and rose from -26.2 to $-48.1 \mathrm{mV}$. The $\mathrm{C}_{7}-\mathrm{OH}$ on the quercetin nucleus also behaves as a polyprotic acid undergoing dissociation at alkaline conditions ( $>\mathrm{pH} 7$ ) by imparting a negative charge. ${ }^{36}$ As the particles become more highly charged, they are likely to be less hydrophobic and less surface-active. ${ }^{36}$ Thus, the $\zeta$ potential results predict that both curcumin and quercetin would be more surface-active at the lower $\mathrm{pH}$. Most notably, curcumin at $\mathrm{pH} 3$ is predicted as the most hydrophobic from the above contact angle measurements, which agrees with the lowest absolute magnitude of $\zeta$-potential in contact with water and also with the population of crystals in the lower size range observed in Figure 2. In other words, curcumin is more easily dispersed in the oil phase (as also noted in the confocal observations discussed below).

W/O Emulsions. Effect of Particle Concentration and $\mathrm{pH}$ on the Stability of W/O Pickering Emulsion Droplets. Water droplet size distributions of the particle-stabilized W/O emulsions are shown at different concentrations of particles (0.06-1.5 wt \%) in Figure 5a,b. In this case, the aqueous phase was adjusted to $\mathrm{pH} 3$. Emulsions prepared at $0.06 \mathrm{wt} \%$ of either curcumin or quercetin particles were not as stable as those with higher concentrations of particles; they tended to coalesce, and the size of the water droplets increased dramatically within 3 days. On the other hand, the size of emulsion droplets prepared at higher concentrations of particles $(>0.14$ wt \%) did not change significantly after 7 


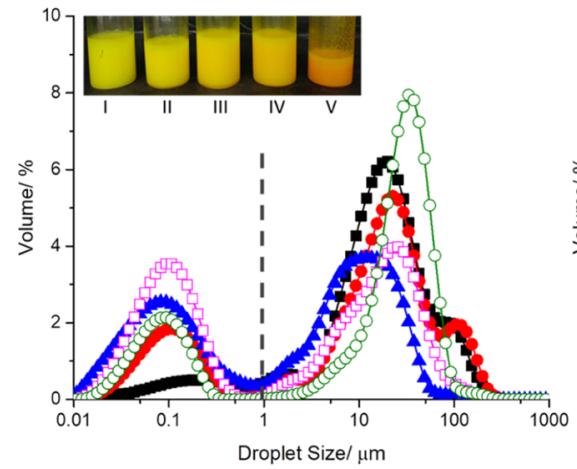

(a)

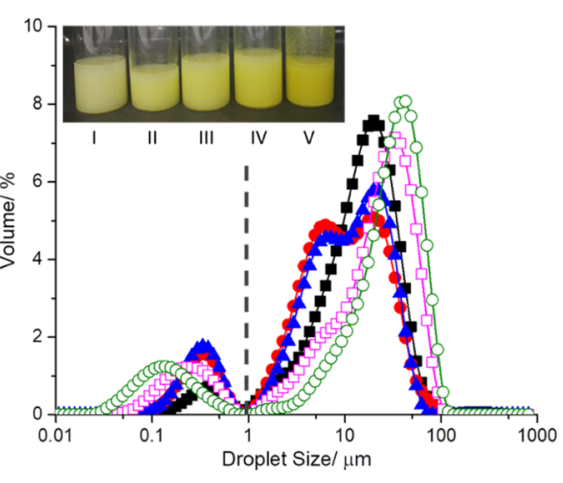

(b)

Figure 5. Mean water droplet size distributions and corresponding visual images of W/O Pickering emulsions (5:95 wt \% w/o ratio) stabilized by curcumin (a) and quercetin (b) crystals at different particle concentrations; 0.06 wt \% [black square] and [I], 0.14 wt \% [red circle] and [II], 0.25 wt \% [blue triangle] and [III], $0.5 \mathrm{wt} \%$ [pink open square] and [IV], $1.5 \mathrm{wt} \%$ [green open circle] and [V] curcumin or quercetin particles at $\mathrm{pH} 3$. The dashed line separates the unabsorbed particles remaining in the continuous phase $(\leq 1 \mu \mathrm{m})$ and water droplets.

days, although some particle sedimentation was observed. The lack of stability of emulsions at $0.06 \mathrm{wt} \%$ of particles suggests incomplete droplet surface coverage by the limited quantity of particles available, leading to coalescence. In the size distribution plots for both sets of emulsions (Figure 5a,b), smaller peaks were observed $(\leq 1 \mu \mathrm{m})$, probably because of the nonadsorbed particles remaining in the continuous phase because the SLS technique cannot distinguish between emulsion droplets and polyphenol particles as scattering centers. $^{37}$ These peaks were more obvious in the curcuminstabilized emulsions, perhaps indicating that curcumin particles were less completely adsorbed at the interface. This was also observed in the confocal images of emulsions (see later). In addition, significant particle sedimentation was observed especially at highest concentrations ( 0.5 and $1.5 \mathrm{wt} \%)$ for both curcumin and quercetin. Higher water volume fraction (10:90 wt \% w/o ratio) was tested. The results show that for both curcumin and quercetin particles, the size of the water droplets was significantly bigger $(20-24 \mu \mathrm{m}$ ) (see Supporting Information, Figure S3) than the size of the water droplets (7$9 \mu \mathrm{m}$ ) at lower volume fraction (5:95 wt \% w/o ratio, Figure 6). In addition, fewer peaks were observed at $\leq 1 \mu \mathrm{m}$ region, indicating that more crystals were absorbed at the interface.

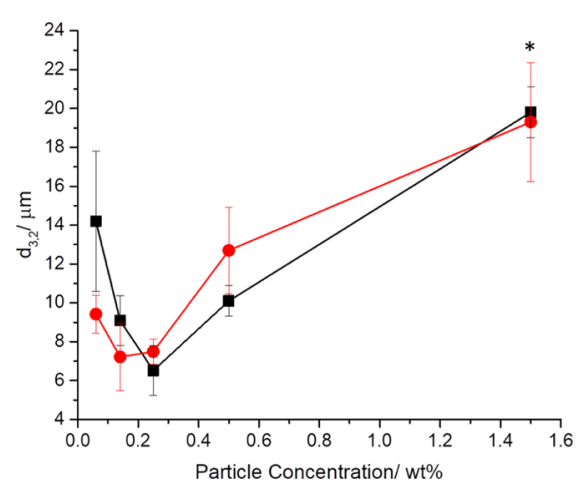

Figure 6. Mean droplet size of water droplets $\left(d_{3,2}\right)$ stabilized by curcumin [black square] or quercetin [red circle] particles at different concentrations: $0.06,0.14,0.25,0.5$, and $1.5 \mathrm{wt} \%$. The $\mathrm{pH}$ of the aqueous phase was adjusted to $\mathrm{pH} 3$. Error bars represent standard deviation of at least three independent experiments. Samples with * do not differ significantly $(p>0.05)$ according to Tukey's test.
As discussed above, some smaller peaks $(\leq 1 \mu \mathrm{m})$ were observed in the size distribution plots (Figure $5 \mathrm{a}, \mathrm{b}$ ) for both curcumin and quercetin particles because of the presence of free particles in the continuous phase (the peaks were separated by a dashed line). Therefore, the actual water droplet size can be identified from the peaks above $1 \mu \mathrm{m}$ for both particle stabilizers and are shown in Figure 6 (the $d_{3,2}$ values were recalculated for water droplets $(>1 \mu \mathrm{m})$ for both systems). Smaller water droplets were identified at the particle concentrations of 0.14 and $0.25 \mathrm{wt} \%$ for quercetin and curcumin, respectively. At a particle concentration below 0.25 wt $\%$ for curcumin and $0.14 \mathrm{wt} \%$ of quercetin, there were not enough particles to fully cover the water droplets. However, at the concentrations indicated above $(0.25$ wt \% for curcumin and 0.14 wt $\%$ of quercetin), there was a maximum surface coverage, and minimum $d_{3,2}$ values were observed. By increasing the concentration of polyphenol particles above 0.25 wt $\%$, the $d_{3,2}$ value started to increase. This reflects the competition between particle adsorption at the interface and particle aggregation in the oil phase as the particle concentration increased. At a very high concentration of particles (1.5 wt \%), the aggregation mechanism in the bulk oil phase appeared to dominate, depleting the particles that could have contributed to the stabilization of the water droplets, otherwise.

W/O Pickering emulsions were also prepared at $\mathrm{pH} 7$. Droplet size distributions for both emulsions (Figure 7a,b) show very similar trends at all particle concentrations with no significant change in the mean size. However, for all the systems, the size of the water droplets increased significantly within 3 days, indicating unstable emulsions. In addition, as observed at $\mathrm{pH} 3$, some smaller peaks below $1 \mu \mathrm{m}$ were seen for both particles (the peaks were separated by a dashed line, and the $d_{3,2}$ values were recalculated for water droplets as above), but the peaks in curcumin-stabilized emulsions were much larger than those in quercetin, again indicating that curcumin was not as efficiently adsorbed to the interface at $\mathrm{pH}$ 7.

Figure $8 \mathrm{a}, \mathrm{b}$ compares the calculated mean droplet sizes (i.e., $>1 \mu \mathrm{m})$ at different particle concentrations and different $\mathrm{pH}$ values. For curcumin-stabilized emulsions with an aqueous phase of $\mathrm{pH} 7$, the mean size of the water droplets was similar at all curcumin concentrations, $d_{3,2}=9-10 \mu \mathrm{m}$. Similarly, quercetin-stabilized emulsions at $\mathrm{pH} 7$ were similar in size at 


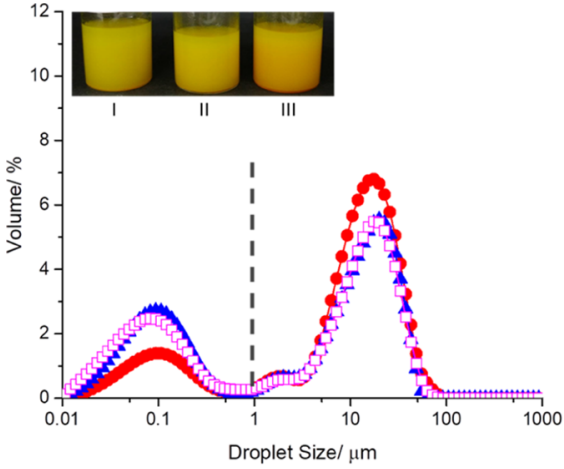

(a)

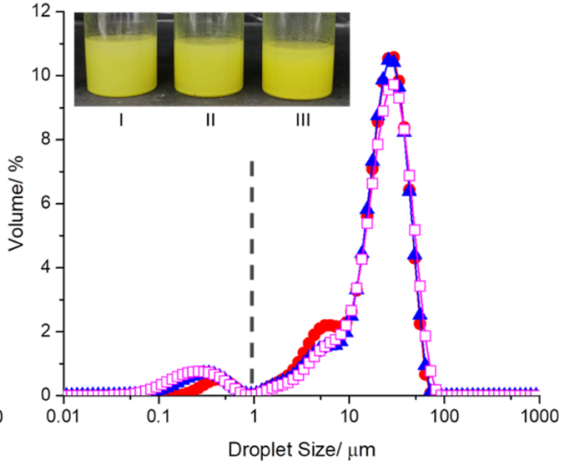

(b)

Figure 7. Mean water droplet size distributions and corresponding visual images of W/O Pickering emulsions (5:95 wt \% w/o ratio) stabilized by curcumin (a) and quercetin (b) crystals at different particle concentrations: $0.14 \mathrm{wt} \%$ [red circle] and [I], $0.25 \mathrm{wt} \%$ [blue triangle] and [II], and $0.5 \mathrm{wt} \%$ [pink open square] and [III] curcumin or quercetin particles at $\mathrm{pH}$ 7. The dashed line separates the unabsorbed particles remaining in the continuous phase $(\leq 1 \mu \mathrm{m})$ and water droplets.

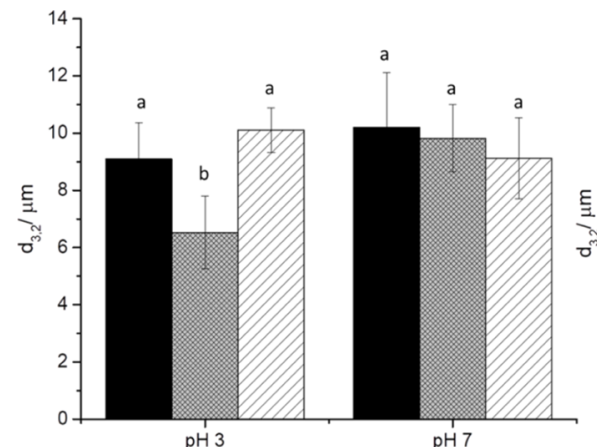

(a)

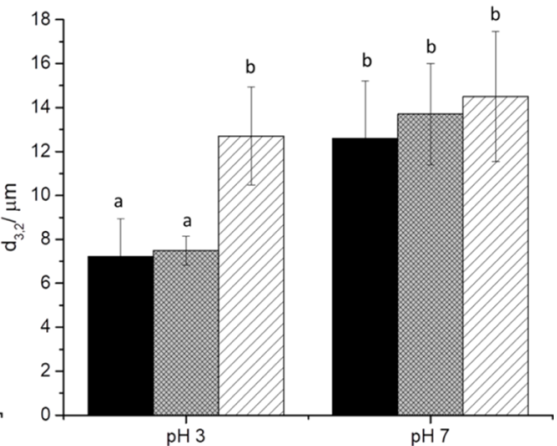

(b)

Figure 8. Mean droplet size $\left(d_{3,2}\right)$ of the W/O Pickering emulsions versus $\mathrm{pH}$ for curcumin (a) and quercetin (b) at different particle concentrations: $0.14 \mathrm{wt} \%$ [black], $0.25 \mathrm{wt} \%$ [gray], and $0.5 \mathrm{wt} \%$ [white]. Error bars represent standard deviation of at least three independent experiments. Bars with the same letter do not differ significantly $(p>0.05)$ according to Tukey's test for each polyphenol crystal at the two different $\mathrm{pH}$ values used.

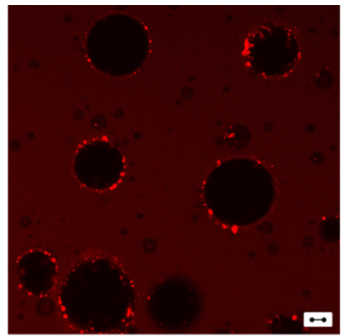

(a)

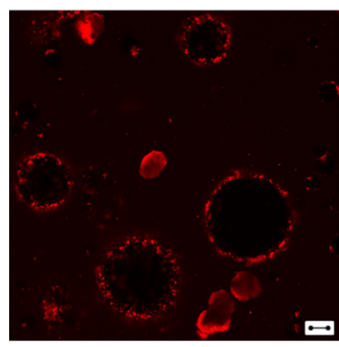

(c)

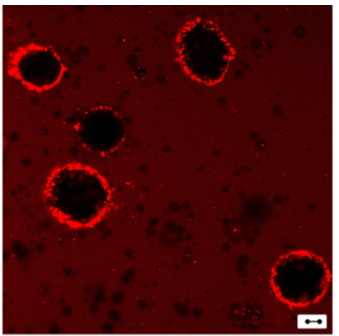

(b)

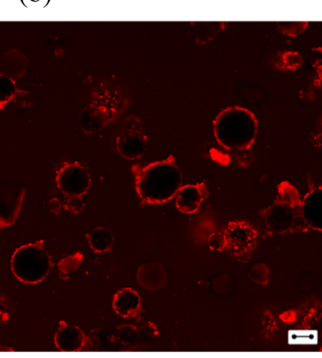

(e)

Figure 9. Confocal images of W/O Pickering emulsions (5:95 wt \% w/o ratio) stabilized by curcumin (red) particles at different concentrations: 0.06 (a), 0.14 (b), 0.25 (c), 0.5 (d), and 1.5 wt \% (e). The $\mathrm{pH}$ of the aqueous phase was adjusted to $\mathrm{pH} 3$. The scale bar is equal to $20 \mu \mathrm{m}$. The brightness in the images is caused by autofluorescence of the curcumin particles ( $488 \mathrm{~nm}$ excitation). 


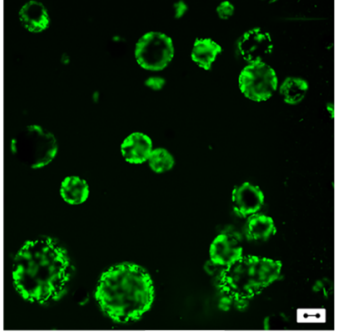

(a)

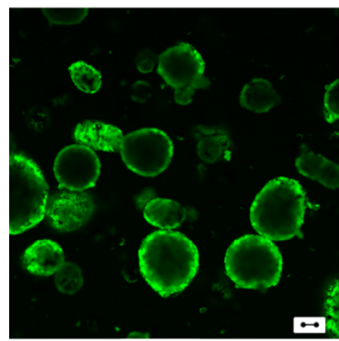

(c)

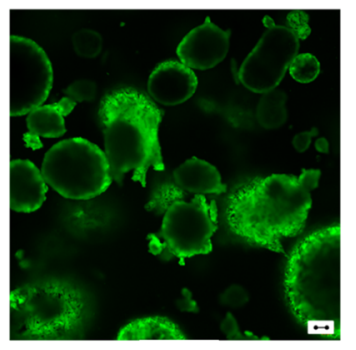

(d)

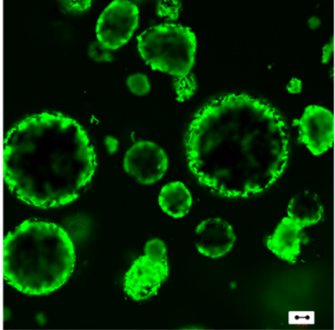

(b)

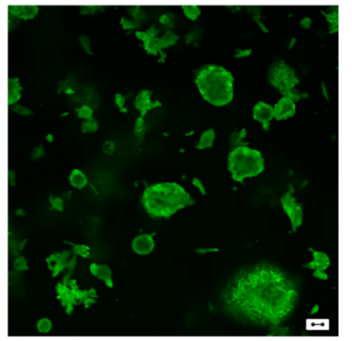

(e)

Figure 10. Confocal images of W/O Pickering emulsions (5:95 wt \% w/o ratio) stabilized by quercetin (green) particles at different concentrations: 0.06 (a), 0.14 (b), 0.25 (c), 0.5 (d), and $1.5 \mathrm{wt} \%$ (e). The $\mathrm{pH}$ of the aqueous phase was adjusted to $\mathrm{pH} 3$. The scale bar is equal to $20 \mu \mathrm{m}$. The brightness in the images is caused by autofluorescence of the quercetin particles (405 nm excitation).

all concentrations, $d_{3,2}=12.5-14.5 \mu \mathrm{m}$. The water droplets stabilized by quercetin particles at $\mathrm{pH} 7$ were larger than those at $\mathrm{pH} 3$ at the concentrations of 0.06 and 0.14 wt \%. Interestingly, at $0.5 \mathrm{wt} \%$ for both particles, the size of water droplets did not vary much on changing the $\mathrm{pH}$. The $\zeta$ potential measurements suggested that both types of particles would have a greater (negative) charge on attaching water at $\mathrm{pH} 7$, which may reduce particle surface activity and increase interparticle repulsion, explaining the decreases in emulsifying capability.

Confocal images of emulsions in Figures 9 and 10 show how water droplets were surrounded by a dense layer of curcumin (red) or quercetin (green) particles, confirming the preferential location of the polyphenol crystals at the W/O interface. The brightness in the images was due to the autofluorescence of the polyphenol particles. Furthermore, some droplets appeared to be not completely spherical, which is another indication of Pickering stabilization. ${ }^{38}$ For curcumin-stabilized emulsions, some small particles appeared in the continuous phase of oil, agreeing with the results from light scattering (Figures 5a and $7 \mathrm{a}$ at $\mathrm{pH} 3$ and 7 , respectively) and macroscopic observations of the emulsions that a significant amount of curcumin particles remained unabsorbed, that is, dispersed in the oil phase. This agrees with the predictions of the contact angle measurements and calculated $\Delta G_{\mathrm{d}}$ discussed above, as well as the particle size distributions in Figure 2. Curcumin is more hydrophobic and more compatible with oil than quercetin, whereas larger crystals of either should have a very large energy barrier to detach from the interface.

To further verify the claim that the curcumin- or quercetinstabilized systems were Pickering emulsions, images were collected at different depths of focus on a single large droplet as shown in Figures 11 and 12. The presence of a continuous bright ring at the $\mathrm{W} / \mathrm{O}$ interface demonstrates even more clearly that water droplets were covered by curcumin or quercetin particles. Moreover, the size and shape of the particles at the interface were assessed by zooming in to a specific region. Quercetin particles had their rodlike shape and

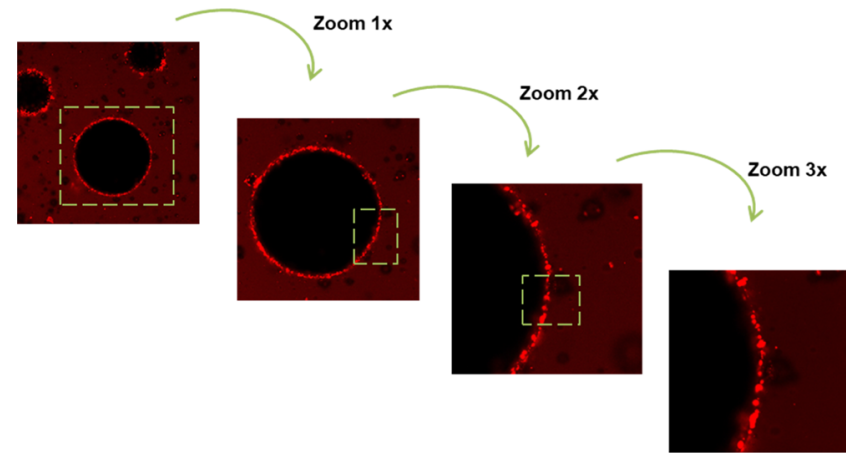

Figure 11. Confocal images of W/O Pickering emulsions stabilized by 0.14 wt $\%$ curcumin particles. The arrows indicate the sequence of images collected at the depths of focus in order to visualize the particles at the $\mathrm{W} / \mathrm{O}$ interface. The brightness in the image is caused by autofluorescence of the curcumin particles ( $488 \mathrm{~nm}$ excitation).

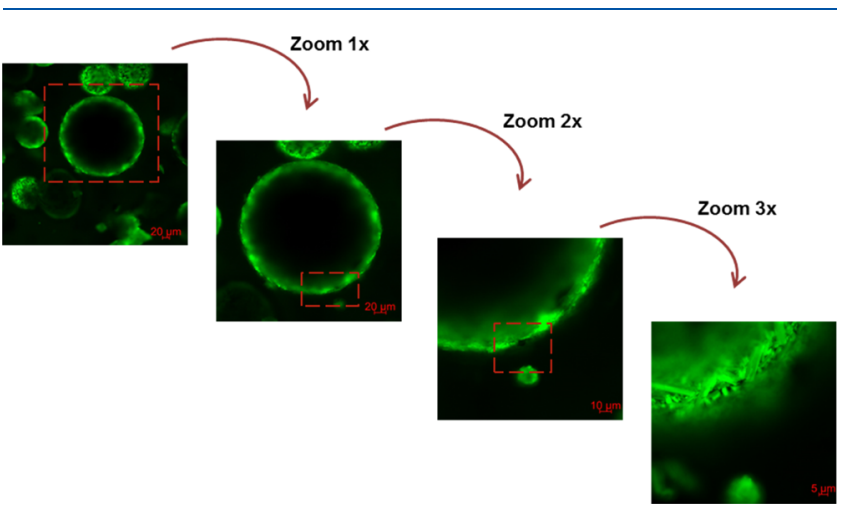

Figure 12. Confocal images of W/O Pickering emulsions stabilized by 0.14 wt $\%$ quercetin particles. The arrows indicate the sequence of images collected at the depths of focus in order to visualize the particles at the $\mathrm{W} / \mathrm{O}$ interface. The brightness in the image is caused by autofluorescence of the quercetin particles ( $405 \mathrm{~nm}$ excitation). 
formed a denser layer at the interface, whereas curcumin particles had their polyhedral shape, in agreement with the images of the particles in oil observed previously (Figures 3 and 4 , respectively).

Interfacial Shear Viscosity $\left(\eta_{i}\right)$. Interfacial shear viscosity is a useful way of monitoring the formation and structuring of adsorbed films. ${ }^{39}$ It gives information about structural and compositional changes within the adsorbed layers and how the surface behavior can be related to the aspects of the formation and stability of emulsions. ${ }^{28,40}$ Figure 13 shows the effect of the

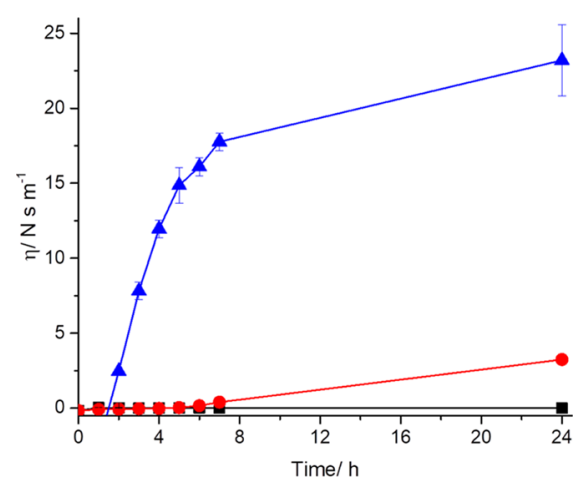

Figure 13. Interfacial shear viscosity at the interface of Milli- $Q$ water with nonpurified oil [black square], nonpurified oil with 0.14 wt $\%$ curcumin particles [red circle], and nonpurified oil with 0.14 wt \% quercetin particles [blue triangle]. The $\mathrm{pH}$ of the aqueous phase was adjusted to $\mathrm{pH}$ 3. Error bars represent standard deviation of at least two independent experiments.

presence of curcumin or quercetin particles dispersed in nonpurified oil on the mean values of interfacial shear viscosity. A control experiment with nonpurified oil and Milli- $\mathrm{Q}$ water ( $\mathrm{pH} 3$ ) was performed, but $\eta_{i}$ was equal with $0 \mathrm{~N} \mathrm{~s} \mathrm{~m}^{-1}$ even after $24 \mathrm{~h}$. Thus, the addition of curcumin or quercetin particles at $0.14 \mathrm{wt} \%$ caused an increase in $\eta_{i}$. In the presence of curcumin particles, the rate of growth of $\eta_{i}$ was very slow at the beginning of the experiment (i.e., up to at least $\approx 7 \mathrm{~h}$ of adsorption), but after $24 \mathrm{~h}, \eta_{i}$ had increased to $1.2 \mathrm{~N} \mathrm{~s} \mathrm{~m}^{-1}$. In contrast, in the presence of quercetin particles at the same concentration, $\eta_{i}$ increased significantly right at the beginning of the experiment and was still increasing even after $24 \mathrm{~h}$ of adsorption ( $>20 \mathrm{~N} \mathrm{~s} \mathrm{~m}^{-1}$ ). This suggests stronger accumulation of quercetin particles at the interface and/or stronger interactions between the particles at the interface compared with curcumin particles. This might be attributed to the larger aspect ratio of the quercetin particles giving higher desorption energy per unit mass and allowing more efficient packing at the interface (Figure 10). The curcumin particles were much smaller $\left(d_{3,2}=0.2 \mu \mathrm{m}\right)$ and polyhedral in shape, giving lower desorption energy per unit mass while at the same time less efficient particle packing at the interface (Figure 9). On the other hand, because both curcumin and quercetin particles are denser than oil, the curcumin particles will also take longer to fall and settle at the interface in the interfacial shear viscosity experiment.

Fat crystals and mono- and diglycerides that are present in vegetable oils can influence the interfacial rheology of the adsorbed particle films. ${ }^{28}$ On the basis of the literature, the coadsorption of mono- and diglycerides tends to destroy high values of $\eta_{i}$ of particle films because they tend to be more surface-active than particles and displace them completely. ${ }^{28}$
For this reason, soybean oil was purified using aluminum oxide in order to remove any surface-active components that may affect the interfacial shear viscosity results. Experiments with curcumin and quercetin particles dispersed in purified oil are shown in the Supporting Information, Figure S4. The same trends were observed in purified oil as with nonpurified oil (Figure 13), indicating that the surface-active materials in the oil did not affect the interfacial viscosity significantly.

\section{CONCLUSIONS}

We have demonstrated for the first time that W/O emulsions can be stabilized with curcumin or quercetin particles, which were both sufficiently hydrophobic according to the contact angle measurements. Microstructural evaluation at various length scales revealed that quercetin particles had a rod shape and larger aspect ratio than curcumin particles, the latter having a polyhedral shape. Neither of particle dispersions significantly suppressed the interfacial tension again, indicating a Pickering stabilization mechanism.

When preparing $\mathrm{W} / \mathrm{O}$ particle-stabilized emulsions, a low concentration of particles ( $0.06 \mathrm{wt} \%)$ was not able to cover the water droplets fully, whereas at a much higher concentration ( 0.5 and $1.5 \mathrm{wt} \%)$, a significant proportion of particles still remained unabsorbed, leading to aggregation of water droplets and particle sedimentation. An optimum concentration of particles with the smallest water droplets and fewer free particles in the continuous phase was identified at 0.14 and 0.25 wt $\%$ for quercetin and curcumin, respectively. Increasing the $\mathrm{pH}$ of the aqueous phase from $\mathrm{pH} 3$ to $\mathrm{pH} 7$ gave more coarse emulsions, possibly related to an increase in the magnitude of the $\zeta$-potential (more negative) of the particle surface when in contact with water. Interfacial shear viscosity results also show stronger film formation at the interface because of stronger interactions between the quercetin particles, possibly because of their larger size and higher aspect ratio. Therefore, we suggest that particle stabilization of $\mathrm{W} / \mathrm{O}$ emulsions may be imparted by biocompatible polyphenol crystals of appropriate size, shape, charge, and wettability. As such, this work should have broad implications in the rational design of stable $\mathrm{W} / \mathrm{O}$ or double emulsions for various soft matter applications, such as food, pharmaceuticals, personal care, agriculture, and so forth, and have implications for the behavior of such polyphenol crystals in food processing and even their digestion. Work is currently ongoing on the process and storage stability of the emulsions.

\section{ASSOCIATED CONTENT}

\section{S Supporting Information}

The Supporting Information is available free of charge on the ACS Publications website at DOI: 10.1021/acs.langmuir.8b01438.

Particle size distribution of curcumin and quercetin crystals passed through all stages of emulsification process; SEM images of curcumin and quercetin dry powders; surface tension measurements; interfacial tension measurements; mean water droplet size distribution of $\mathrm{W} / \mathrm{O}$ emulsions at 10:90 wt \% w/o ratio; and interfacial shear viscosity using purified oil (PDF) 


\section{AUTHOR INFORMATION}

\section{Corresponding Author}

*E-mail: A.Sarkar@leeds.ac.uk.

\section{ORCID}

Anwesha Sarkar: 0000-0003-1742-2122

\section{Notes}

The authors declare no competing financial interest.

\section{ACKNOWLEDGMENTS}

The authors gratefully acknowledge the Engineering and Physical Sciences Research Council funded Centre for Doctoral Training in Soft Matter and Functional Interfaces, grant ref. no. EP/L015536/1 as well as Nestlé PTC Confectionery (York, UK) for the financial support. The authors would like to thank Dr Alexander Kulak from the School of Chemistry at the University of Leeds for assistance with the SEM images.

\section{REFERENCES}

(1) Lam, S.; Velikov, K. P.; Velev, O. D. Pickering stabilization of foams and emulsions with particles of biological origin. Curr. Opin. Colloid Interface Sci. 2014, 19, 490-500.

(2) Xiao, J.; Li, Y.; Huang, Q. Recent advances on food-grade particles stabilized Pickering emulsions: Fabrication, characterization and research trends. Trends Food Sci. Technol. 2016, 55, 48-60.

(3) Pickering, S. U. Cxcvi.-emulsions. J. Chem. Soc., Dalton Trans. 1907, 91, 2001-2021.

(4) Dickinson, E. Use of nanoparticles and microparticles in the formation and stabilization of food emulsions. Trends Food Sci. Technol. 2012, 24, 4-12.

(5) Sarkar, A.; Murray, B.; Holmes, M.; Ettelaie, R.; Abdalla, A.; Yang, $\mathrm{X}$. In vitro digestion of Pickering emulsions stabilized by soft whey protein microgel particles: influence of thermal treatment. Soft Matter 2016, 12, 3558-3569.

(6) Luo, Z.; Murray, B. S.; Yusoff, A.; Morgan, M. R. A.; Povey, M. J. W.; Day, A. J. Particle-Stabilizing Effects of Flavonoids at the OilWater Interface. J. Agric. Food Chem. 2011, 59, 2636-2645.

(7) Binks, B. P. Particles as surfactants-similarities and differences. Curr. Opin. Colloid Interface Sci. 2002, 7, 21-41.

(8) Binks, B. P.; Lumsdon, S. O. Influence of Particle Wettability on the Type and Stability of Surfactant-Free Emulsions. Langmuir 2000, $16,8622-8631$.

(9) Hunter, T. N.; Pugh, R. J.; Franks, G. V.; Jameson, G. J. The role of particles in stabilising foams and emulsions. Adv. Colloid Interface Sci. 2008, 137, 57-81.

(10) Aveyard, R.; Binks, B. P.; Clint, J. H. Emulsions stabilised solely by colloidal particles. Adv. Colloid Interface Sci. 2003, 100-102, 503546.

(11) Binks, B. P.; Horozov, T. S. Colloidal Particles at Liquid Interfaces; Cambridge University Press, 2006.

(12) Kim, J.-H.; Kim, S.-I.; Kyong, K.-Y.; Lee, E.-J.; Cho, W.-G.; Yoon, M.-S. Effect of aqueous phase composition on the stability of a silica-stabilized water-in-oil emulsion. J. Soc. Cosm. Sci. Korea 2004, 30, 353-359.

(13) Midmore, B. R. Effect of aqueous phase composition on the properties of a silica-stabilized W/O emulsion. J. Colloid Interface Sci. 1999, 213, 352-359.

(14) Skelhon, T. S.; Grossiord, N.; Morgan, A. R.; Bon, S. A. F. Quiescent water-in-oil Pickering emulsions as a route toward healthier fruit juice infused chocolate confectionary. J. Mater. Chem. 2012, 22, 19289-19295.

(15) Rein, D. M.; Khalfin, R.; Cohen, Y. Cellulose as a novel amphiphilic coating for oil-in-water and water-in-oil dispersions. J. Colloid Interface Sci. 2012, 386, 456-463.
(16) Binks, B. P.; Lumsdon, S. O. Pickering emulsions stabilized by monodisperse latex particles: effects of particle size. Langmuir 2001, $17,4540-4547$.

(17) Brugger, B.; Rosen, B. A.; Richtering, W. Microgels as stimuliresponsive stabilizers for emulsions. Langmuir 2008, 24, 1220212208.

(18) Bordenave, N.; Hamaker, B. R.; Ferruzzi, M. G. Nature and consequences of non-covalent interactions between flavonoids and macronutrients in foods. Food Funct. 2014, 5, 18-34.

(19) Tsao, R. Chemistry and biochemistry of dietary polyphenols. Nutrients 2010, 2, 1231-1246.

(20) Sharma, R. A.; Gescher, A. J.; Steward, W. P. Curcumin: the story so far. Eur. J. Cancer 2005, 41, 1955-1968.

(21) Araiza-Calahorra, A.; Akhtar, M.; Sarkar, A. Recent advances in emulsion-based delivery approaches for curcumin: From encapsulation to bioaccessibility. Trends Food Sci. Technol. 2018, 71, 155-169.

(22) Payton, F.; Sandusky, P.; Alworth, W. L. NMR study of the solution structure of curcumin. J. Nat. Prod. 2007, 70, 143-146.

(23) Formica, J. V.; Regelson, W. Review of the biology of quercetin and related bioflavonoids. Food Chem. Toxicol. 1995, 33, 1061-1080.

(24) Cretu, R.; Dima, C.; Bahrim, G.; Dima, S. Improved solubilization of curcumin with a microemulsification formulation. The Annals of the University of Dunarea De Jos of Galati. Fascicle VI. Food Technology, 2011; Vol. 35(2), p 46.

(25) Roedig-Penman, A.; Gordon, M. H. Antioxidant properties of myricetin and quercetin in oil and emulsions. J. Am. Oil Chem. Soc. 1998, 75, 169-180.

(26) Burgaud, I.; Dickinson, E.; Nelson, P. V. An improved highpressure homogenizer for making fine emulsions on a small scale. Int J. Food Sci. Technol. 1990, 25, 39-46.

(27) Niknafs, N. Mechanistic Understanding of Emulsion Formation during Processing; University of Birmingham, 2011.

(28) Murray, B. S. Interfacial rheology of food emulsifiers and proteins. Curr. Opin. Colloid Interface Sci. 2002, 7, 426-431.

(29) Sarkar, A.; Zhang, S.; Murray, B.; Russell, J. A.; Boxal, S. Modulating in vitro gastric digestion of emulsions using composite whey protein-cellulose nanocrystal interfaces. Colloids Surf., B 2017, $158,137-146$.

(30) Duffus, L. J.; Norton, J. E.; Smith, P.; Norton, I. T.; Spyropoulos, F. A comparative study on the capacity of a range of food-grade particles to form stable $\mathrm{O} / \mathrm{W}$ and $\mathrm{W} / \mathrm{O}$ Pickering emulsions. J. Colloid Interface Sci. 2016, 473, 9-21.

(31) Tønnesen, H. H.; Másson, M.; Loftsson, T. Studies of curcumin and curcuminoids. XXVII. Cyclodextrin complexation: solubility, chemical and photochemical stability. Int. J. Pharm. 2002, 244, 127135 .

(32) Balasubramanian, K. Molecular orbital basis for yellow curry spice curcumin's prevention of Alzheimer's disease. J. Agric. Food Chem. 2006, 54, 3512-3520.

(33) Rossi, M.; Rickles, L. F.; Halpin, W. A. The crystal and molecular structure of quercetin: A biologically active and naturally occurring flavonoid. Bioorg. Chem. 1986, 14, 55-69.

(34) Al-Rossies, A. A. S.; Al-Anazi, B. D.; Paiaman, A. M. Effect of $\mathrm{pH}$-values on the contact angle and interfacial tension. Nafta 2010, $61,181-186$

(35) Melle, S.; Lask, M.; Fuller, G. G. Pickering emulsions with controllable stability. Langmuir 2005, 21, 2158-2162.

(36) Luo, Z.; Murray, B. S.; Ross, A.-L.; Povey, M. J. W.; Morgan, M. R. A.; Day, A. J. Effects of $\mathrm{pH}$ on the ability of flavonoids to act as Pickering emulsion stabilizers. Colloids Surf., B 2012, 92, 84-90.

(37) Atarés, L.; Marshall, L. J.; Akhtar, M.; Murray, B. S. Structure and oxidative stability of oil in water emulsions as affected by rutin and homogenization procedure. Food Chem. 2012, 134, 1418-1424.

(38) Dickinson, E. Food emulsions and foams: Stabilization by particles. Curr. Opin. Colloid Interface Sci. 2010, 15, 40-49.

(39) Burke, J.; Cox, A.; Petkov, J.; Murray, B. S. Interfacial rheology and stability of air bubbles stabilized by mixtures of hydrophobin and $\beta$-casein. Food Hydrocolloids 2014, 34, 119-127. 
(40) Murray, B. S.; Dickinson, E. Interfacial rheology and the dynamic properties of adsorbed films of food proteins and surfactants. Food Sci. Technol. Int. 1996, 2, 131-145. 